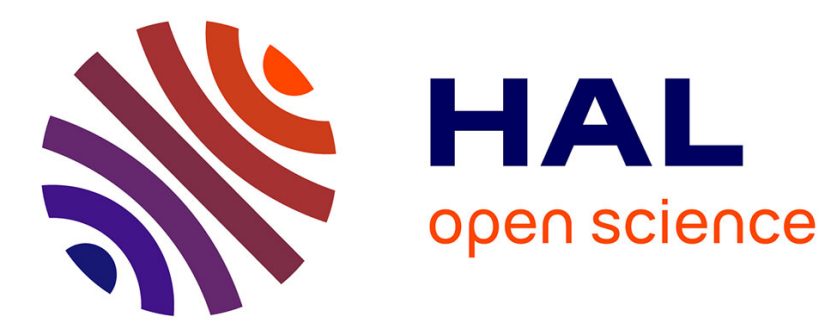

\title{
Phenotypical characterization of children with hypersensitivity reactions to NSAIDs
}

\author{
Mathias Cousin, Anca Chiriac, Nicolas Molinari, Pascal Demoly, Davide
}

Caimmi

\section{- To cite this version:}

Mathias Cousin, Anca Chiriac, Nicolas Molinari, Pascal Demoly, Davide Caimmi. Phenotypical characterization of children with hypersensitivity reactions to NSAIDs. Pediatric Allergy and Immunology, 2016, 27 (7), pp.743-748. 10.1111/pai.12596 . hal-01361516

\section{HAL Id: hal-01361516 https://hal.sorbonne-universite.fr/hal-01361516}

Submitted on 7 Sep 2016

HAL is a multi-disciplinary open access archive for the deposit and dissemination of scientific research documents, whether they are published or not. The documents may come from teaching and research institutions in France or abroad, or from public or private research centers.
L'archive ouverte pluridisciplinaire HAL, est destinée au dépôt et à la diffusion de documents scientifiques de niveau recherche, publiés ou non, émanant des établissements d'enseignement et de recherche français ou étrangers, des laboratoires publics ou privés. 
1 Phenotypical characterization of children with hypersensitivity reactions to NSAIDs

2

3 Authors: Cousin Mathias, $\mathrm{MD}^{1}$, Chiriac Anca, $\mathrm{MD}^{1,2}$, Molinari Nicolas, $\mathrm{PhD}^{3}$, Demoly Pascal,

$4 \quad \mathrm{PhD}^{1,2}$, Caimmi Davide, $\mathrm{MD}^{1,2}$

5

6 Affiliations:

$7 \quad{ }^{1}$ Unité d'allergologie, Département de Pneumologie et Addictologie, Hôpital Arnaud de Villeneuve,

8 CHRU de Montpellier, France

$9{ }^{2}$ Sorbonne Universités, UPMC Paris 06, UMR-S 1136, IPLESP, Equipe EPAR, F-75013 Paris,

10 France

$11{ }^{3}$ Département de Statistiques, IMAG UMR5149 S, CHRU de Montpellier, Montpellier, France 12

13 Running title: NSAIDs hypersensitivity in Pediatrics

14

15 Corresponding Author: Davide Caimmi, Service d'allergologie, CHRU de Montpellier, Hôpital 16 Arnaud de Villeneuve, 371, Av. du Doyen Gaston Giraud, 34295 Montpellier CEDEX 5, tel :

17 +33630061134, fax : +33467330740, e-mail : davide.caimmi@gmail.com

18

19 Word Count: 2499 words

$20 \quad 4$ Tables 
Abstract

Cousin M, Chiriac A, Molinari N, Demoly P, Caimmi D.

Phenotypical characterization of children with hypersensitivity reactions to NSAIDs

Pediatr Allergy Immunol

Background. Non-steroidal anti-inflammatory drugs (NSAIDs) are the main cause of drug-induced hypersensitivity in children. Many classifications have been proposed, focusing on adults. So far, no large study has deeply investigated a pediatric cohort. The aim of the present study was to describe a population of NSAID hypersensitive patients reporting a reaction during their childhood, and to verify if it is possible to classify pediatric patients, following the EAACI/ENDA classification.

Methods. We conducted a historical-prospective study including patients evaluated from 1996 to 2015 in the allergy unit of the Montpellier University Hospital. We included 635 patients. For each patient, we recorded clinical manifestations and possible co-morbidities, and tried to identify possible risk factors.

Results. NSAIDs hypersensitivity was diagnosed in 107 out of 635 patients (16.9\%). In this group, 43 patients $(40.2 \%)$ could not be classified following the ENDA recommendations. The main discrepancies were on the patients' clinical manifestations and on their possible underlying diseases. We identified, on a multivariate analysis, some risk factors for NSAID hypersensitivity: chronic urticaria (OR 7.737, 3.375-18.296 95\%CI), atopic status (OR 2.514, 1.504-4.364 95\%CI) and allergic rhino-conjunctivitis (OR 1.799, 1.138-2.837 95\%CI). On the basis of our results, we are proposing an adapted classification for NSAIDs hypersensitivity in children.

Conclusions. The current ENDA classification does not seem to be adapted for pediatric patients; a modified version does. Our study could help allergists better understand the differences between adults and children in developing hypersensitivity reactions to NSAIDs but further large-scale prospective longitudinal analyses are required to validate this new classification.

Key Words: drug hypersensitivity, NSAIDs, children, classification, drug allergy.

The Authors have no conflict of interest to declare for the present paper. 


\section{Introduction}

52 Non-steroidal anti-inflammatory drugs (NSAIDs) are widely used to treat fever and pain in

53 children. Nevertheless, they are currently considered as the first cause of drug-induced

54 hypersensitivity (HS) reactions in children $(1,2)$. Two different mechanisms are able to elicit HS reactions: immunological mechanisms responsible for allergic HS reactions (such as IgE and T-cells mediated reactions); and non-immunologic ones (mainly due to cyclooxygenases inhibition). Several classifications have been proposed for NSAIDs HS, but no large study has so far tried to classify NSAIDs HS focusing only on a pediatric population.

In 2001, Stevenson classified patients according to their clinical history but this classification did not take into account the reactions recorded during drug provocation tests (DPTs) (3). Thereafter, Quiralte proposed a classification with seven groups according to the patient's underlying disease (4). The most recent NSAIDs HS classification highlighted five groups of patients, and has been published by the European Network for Drug Allergy (ENDA) group, an EAACI drug allergy Interest Group (5). This classification was based on the authors' expertise in hypersensitivity reactions to NSAIDs. The publication does not differentiate between age groups, and it has to be underlined that most of the expertise comes from adolescents and adults. Since its publication, clinical observations and literature data (6) reported that some patients diagnosed with NSAIDs HS could not be classified into the possible phenotypes described by the ENDA group (5). Moreover, while the Widal's syndrome (or Samter's triad) is used as a reference phenotype for the first group of the ENDA classification, "NSAID's-exacerbated respiratory disease", it is known that such a syndrome has never been described before the age of 13 (7). In addition, a recent paper (1) reported that the association of cutaneous and respiratory symptoms is frequently observed in children with NSAIDs HS, which is a combination of different phenotypes proposed by the current classification, but is not highlighted as a single group.

Considering the hypothesis that HS phenotypes may differ between the adult and pediatric population, the aim of the present study was to describe a population of children with a diagnosis of 
NSAIDs HS, and to try to classify them on the basis of the current ENDA recommendation. We also tried to identify possible risk factors for NSAIDs HS in children, and to propose a pediatric version of the ENDA classification.

\section{Methods}

\section{Patients included}

We conducted a historical-prospective study including all patients evaluated at the allergy unit of the University Hospital of Montpellier (France) for a clinical history of possible NSAIDs HS, which occurred during the pediatric age, from September 1996 to July 2015, and who underwent an oral DPT. Following the diagnostic work-up recommendations for NSAIDs HS (8), we classified patients on the basis of the clinical reaction reported and the one recorded at the time of the DPT; the different chemical classes of NSAIDs involved in their reaction; the presence of an underlying chronic disease (asthma, chronic urticaria, chronic rhinosinusitis, atopy as defined by the presence of at least one positive skin prick test to the common aeroallergens of Montpellier area); the delay of reaction; and the suspected HS mechanism.

Clinical data were collected through the validated ENDA drug allergy questionnaire (9). DPTs were performed under strict hospital surveillance at least four weeks after the last patient's HS reaction, according to the unit's protocol $(5,10)$. Patients had not been taking any $\mathrm{H} 1$-antihistamines or other drug possibly affecting the results of the test. Patients on B-blockers were asked to visit their specialist to stop the drug 2 days prior to the test. Administration was single-blinded and performed on the ward by a physician with full resuscitation back up. In case of a possible positive DPT, another molecule would be tested to find a safe alternative for the patient, at least 4 to 6 weeks after the previous DPT. All data were collected in the Drug Allergy and Hypersensitivity Database $\left(\mathrm{DAHD}^{\circledR}\right)$ using FileMaker Pro 13 software. 
103 We excluded all patients who had experienced severe life-threatening skin reactions (toxic 104 epidermal necrolysis and Stevens Johnson syndrome), drug-induced autoimmune diseases or severe 105 organ involvements (such as cytopenias, hepatitis, DRESS-Drug Reaction with Eosinophilia and 106 Systemic Symptoms), since DPTs are contra-indicated for such reactions. All patients refusing 107 DPTs were excluded as well. Written consent was obtained before the test after informing the 108 patients and the parents of children.

\section{Clinical data}

111 For each patient, we looked for the following possible clinical entities, occurring both at the time of 112 the reaction and of the DPT: urticaria, angioedema (AO), maculopapular eruption, fixed drug 113 eruption, rhinitis, conjunctivitis, bronchospasm (defined by dyspnea associated with cough and/or 114 wheezing and/or a fall of $20 \%$ of the patient's basal FEV1 value), ENT-related dyspnea, 115 psychogenic dyspnea (diagnosis per exclusionem), gastrointestinal signs, anaphylaxis. Anaphylaxis 116 was defined as a rapid allergic response involving two different organs with or without associated 117 hypotension (11). Laryngeal angioedema associated dyspnea was considered as a muco-cutaneous 118 symptom.

119 We tested children with the culprit drug and, in case of a positive DPT, patients were tested, at least 1204 weeks after the previous DPT, with a different NSAID, belonging to another chemical subgroup. 121 All together, 14 different NSAIDs have been tested, belonging to different chemical subgroups: 122 acetylsalicylic acid, celocoxib, diclofenac, etoricoxib, ibuprofen, ketoprofen, meloxicam, 123 metamizole, niflumic acid, nimesulide, piroxicam, trofecoxib, tiaprofenic acid. 124 Paracetamol/acetaminophen was included as well in the list of the 14 tested drugs.

\section{Patients' classification}

127 In order to evaluate the accuracy of the ENDA classification (Table 1) in our pediatric cohort, we 128 tried to attribute each patient to the different ENDA groups, numbered from 1 to 5: 
1. NSAIDs-exacerbated respiratory disease

2. NSAIDs-exacerbated cutaneous disease

3. NSAIDs-induced urticaria/angioedema

4. Single-NSAID-induced urticaria/angioedema or anaphylaxis

5. Single-NSAID-induced delayed reactions

134 Whenever a phenotype did not strictly correspond to one of the 5 groups, the patient was classified as "divergent" according to the following criteria:

- the patient was included in the group that seemed the closest to his phenotype;

- we used a letter to highlight for which feature of the group the patient did not properly fit in the ENDA classification: A - clinical manifestations; $\mathrm{B}$ - delay of the reaction; $\mathrm{C}$ underlying disease; D - cross-reactivity (for example, an asthmatic patient who developed urticaria to several NSAIDs was classified as 3C);

confidence interval. A multivariate analysis was conducted using a logistic regression model to determine independent risk factors of NSAIDs, in which we included all variables associated with a $\mathrm{p}$ value below 0.20 in the univariate analysis. Then, a stepwise procedure allowed obtaining the final multivariate model. We considered as statistically significant a p-value $<0.05$.

\section{Results}


635 patients fulfilled our inclusion criteria. In 107 out of 635 (16.9\%) a reaction was recorded through the DPT, and a diagnosis of NSAIDs HS was reached. 62 out of 107 patients were female (57.9\%). The characteristics of the age distribution of our patients are shown in Table 2.

In the group of the 107 diagnosed patients, 77 (72.0\%) were atopic and 47 (43.9\%) were sensitized to house dust mites. We are missing these data in 8 patients, that were therefore considered as nonatopic nor sensitized to mites. Also, 19 patients (17.8\%) suffered from chronic urticaria, 45 (42.1\%) reported symptoms of allergic rhino-conjunctivitis, and 37 (34.6\%) were asthmatic. Two of the asthmatic patients, who were nevertheless adults at the time of their first DPT, presented with nasal polyposis as well. Other allergic diseases included atopic dermatitis (7 patients, $6.5 \%$ ), food allergy (6 patients, 5.6\%), chronic rhino-sinusitis (2 patients, 1.9\%), other drug allergies (2 patients, $1.9 \%$ ), and hymenoptera venom allergy (1 patient, $0.9 \%)$.

On a multivariate analysis, four risk factors were highlighted for NSAIDs HS: urticaria (adjusted OR 7.737, 3.375-18.296, 95\%CI, p-value <0.001), atopy (adjusted OR 2.514, 1.504-4.364 95\%CI, p-value <0.001), allergic rhino-conjunctivitis (adjusted OR 1.799, 1.138-2.837 95\%CI, p-value 0.011), and the age at the first reaction (adjusted OR 1.083, 1.033-1.137 95\%CI, $p$-value $<0.001$ ). In the group of the 107 patients, we ran a total of 192 DPT: 46 patients (43.0\%) only took one, and they did not come back to find a safe alternative, 44 (41.1\%) underwent 2 DPT, 12 patients (11.2\%) were tested for 3 different molecules, $3(2.8 \%)$ for 4 , and 2 patients (1.9\%) came back 5 times to be tested.

Overall clinical symptoms presented by patients during their reported reaction and during DPT were: urticaria/angioedema (104 patients, 97.2\%), bronchospasm (33 patients, 30.8\%), conjunctivitis (31 patients, 29.0\%), anaphylaxis (27 patients, 25.2\%), ENT-related dyspnea (27 patients, 25.2\%), maculopapular eruption (11 patients, 10.3\%), rhinitis (8 patients, $7.5 \%$ ), and gastro-intestinal symptoms (1 patient, $0.9 \%)$.

\section{Classification of hypersensitive patients, according to the ENDA classification}


Among the 107 patients with NSAIDs HS, 43 patients (40.2\%) could not be completely classified in the current ENDA classification and were divergent for one criterion, while 4 patients (3.7\%) were considered as "not possible to classify" (divergent for more than one criterion), as shown in Table 3. Patients were divergent mainly for the feature A, "clinical manifestation" and C, "underlying disease". No patient was divergent for the feature B, "delay of reaction". No patient strictly corresponded to the group 1, "NSAIDs-exacerbated respiratory disease" of the ENDA classification (Table 3).

Among the 22 patients belonging to the group 4, "single-NSAID-induced urticaria/angioedema or anaphylaxis", the main culprit drugs were paracetamol/acetaminophen ( 7 patients, $31.8 \%$ of the group) and ibuprofene (7 patients, $31.8 \%$ of the group). All these patients had a negative DPT to at least one other NSAID. The rest of the results are shown in Table 3.

\section{New possible pediatric classification}

On the basis of our results, we proposed a new classification in three groups, strictly derived from the ENDA one. This classification remains focused on the pathophysiologic HS mechanism, but we also included, as a feature, the impact of risk factors (urticarial, atopy, allergic rhino-conjunctivitis, and age at first reaction). We excluded the presence of underlying diseases (which could occur later in life), and modified the possible clinical manifestations (Table 4).

In this new classification, all our patients could be classified and no patient was divergent. We included 91 patients $(85.1 \%)$ in the group I, "non-allergic NSAIDs hypersensitivity", 15 patients $(14.0 \%)$ in the group II, "single NSAID-induced urticarial/angioedema/anaphylaxis", and 1 patient $(0.9 \%)$ in the group III, "single NSAID-induced delayed reactions". The group I contains actually groups 1 through 3 of the ENDA classification, and it has to be considered as a transitory group, since patients may develop underlying chronic diseases throughout age. Even though a recent paper did not highlight a correlation between NSAID-induced urticaria and evolution toward chronic urticaria (12), these data refer to adult patients over a 12-years follow-up, and might differ from 
what we might record in a strictly pediatric population. Therefore, so far, the evolution of the

208 immune system and the possible appearance of chronic diseases seem not to allow, in children, a 209 differentiation between the first 3 ENDA groups.

212 Discussion

213 Drug hypersensitivity is acquiring more and more importance in patients' everyday life. The 214 EAACI and the ENDA group in particular have proposed several protocols and tools to guide 215 allergists to perform a correct diagnosis and a complete allergy work-up in patients presenting with 216 a suspected hypersensitivity reaction to drugs. As for NSAIDs, a classification has been proposed in 217 2013, but it focused on adult populations. The fact that it is now clear that NSAIDs HS is a key 218 issue in the pediatric population as well, made it imperative to evaluate the appropriateness of such 219 a classification in this group of patients.

220 To our knowledge, we described the largest pediatric cohort with a diagnosis of NSAIDs 221 hypersensitivity. The first aspect highlighted by our results is that the ENDA classification did not 222 allow us to classify more than $40 \%$ of the patients, and it seems therefore clear that the current 223 recommendations are not suitable for the pediatric population.

224 According to a recent pediatric study by Cavkaytar et al. (13), who analyzed a cohort of 30 NSAIDs hypersensitive patients, phenotypes are different in children and adults and pediatric subjects cannot 226 completely be classified in the latest classifications $(5,6)$. In the same study, $27 \%$ of patients were 227 diagnosed as NSAID hypersensitive and only one patient was classified in the group "NSAIDs228 exacerbated respiratory disease" of ENDA classification (13). Our results are similar to those 229 highlighted by Cavkaytar et al: in fact, the main problem we found in our cohort was related to the 230 first group proposed by ENDA, "NSAIDs-exacerbated respiratory disease", since none of our 231 patients could perfectly fit in this group. Indeed, this group was initially created for adults suffering 232 from late onset asthma, which is frequently associated with chronic rhino-sinusitis and nasal 
233 polyposis and with a possible progression towards severe asthma (recurrent respiratory 234 exacerbations and corticosteroid-dependence) $(14,15)$.

235 Considering that NSAIDs HS, and drug HS in general, tends to persist over the years, we could 236 speculate that the evolution of children's immune system and the possible appearance of new and 237 persistent underlying diseases make it difficult to include patients in this group, even though the 238 same patients may belong to it once grown. Nevertheless, in a pediatric classification, it seems 239 inappropriate to classify children according to their underlying disease.

240 An interesting aspect of our results is that most of our patients (97.2\%) reacted to NSAIDs 241 presenting urticaria and/or angioedema as a symptom. Therefore, mucocutaneous symptoms are not 242 specific during the pediatric age and not sufficient to differentiate and classify patients. Also, only 243 very few patients (1.9\%) presented isolated bronchospasm as a symptom, and such a result is 244 confirmed by other authors $(2,16)$.

245 In the literature, physical exercise is often considered as a risk factor to develop an HS reaction to 246 NSAIDs; also, NSAIDs are known as a potential trigger for developing food allergy reactions (1). 247 However, recent NSAIDs HS classification did not include these risk factors. We did not find a 248 correlation between food allergy and NSAIDs HS, and we did not have patients who presented a 249 pediatric clinical history in which physical exercise was related to the appearance of HS symptoms 250 after NSAID intake (data not shown). In the literature, a significant association has been found 251 between house dust mites' sensitization and NASIDs hypersensitivity (14). In our study, atopy (but 252 not particularly house dust mites sensitization), urticaria, allergic rhino-conjunctivitis, and an early 253 reaction in life were found as significant risk factors for hypersensitivity reactions to NSAIDs.

254 Taking into account all of these considerations, we proposed a new classification for NSAIDs HS in 255 children, in which we included the presence of the previously mentioned risk factors, and we 256 neglected the presence of possible underlying chronic diseases. This classification could actually be 257 a helpful tool to understand the mechanisms leading to the HS reaction and to guide allergists in 258 their work-up. 
259 A limitation of this study was that we didn't perform any skin test for the patients who presented 260 anaphylactic reaction or reaction to a single NSAID in their clinical history. Such data could help 261 proving the possible IgE mediated mechanism. Nevertheless, skin tests are not standardized nor 262 validated in clinical practice for NSAIDs and, more importantly, we preferred to use DPT as the 263 gold standard to reach a diagnose of hypersensitivity reaction. Also, we did not test many patients 264 with aspirin. This is due to the fact that such a drug is rarely prescribed in children, due to the risk 265 of Reye's syndrome, and, therefore, a clinical approach to NSAIDs hypersensitivity in children 266 cannot focus on such molecule, which, on the other hand, is often administered in the adult 267 population. Another possible limitation of the present study concerns the diagnosis of rhino-sinusitis 268 and nasal polyposis. Indeed, these diagnoses are mostly done in adult patients and require 269 endoscopic and/or imaging techniques. Therefore, we could not exclude an underestimation of these 270 diseases, even though, at least for nasal polyposis, such a diagnosis is rare during childhood and 271 generally related to other diseases.

272 In conclusion, during childhood every child is exposed at least once to a NSAID, and there are no 273 alternative, on a routine base, to treat fever and/or pain. An accurate classification of NSAIDs 274 hypersensitive patients could help better understand the pathology of these reactions and guide the 275 management of these patients since their early age. We believe that our study could help allergists 276 better understand the differences between adults and children in developing HS reactions to 277 NSAIDs and could indicate a possible way to modify the present classification. 
1. Blanca-Lopez,N, Cornejo-García JA, Plaza-Serón MC et al. Hypersensitivity to nonsteroidal anti-inflammatory drugs in children and adolescents: cross-Intolerance reactions. J Investig Allergol Clin Immunol 2015: 25: 259-69.

2. Liew WK, Chiang WC, Goh AE. Paediatric anaphylaxis in a Singaporean children cohort: changing food allergy triggers over time. Asia Pac Allergy 2013: 3: 29-34.

3. Stevenson DD, Sanchez-Borges M, Szczeklik A. Classification of allergic and pseudoallergic reactions to drugs that inhibit cyclooxygenase enzymes. Ann Allergy Asthma Immunol 2001: 87: 177-80.

4. Quiralte J, Blanco C, Delgado J et al. Challenge-based clinical patterns of 223 Spanish patients with nonsteroidal anti-inflammatory-drug-induced-reactions. J Investig Allergol Clin Immunol 2007: 17: 182-8.

5. Kowalski ML, Asero R, Bavbek $\mathrm{S}$ et al. Classification and practical approach to the diagnosis and management of hypersensitivity to nonsteroidal anti-inflammatory drugs. Allergy 2013: 68: 1219-32.

6. Caimmi S, Caimmi D, Bousquet PJ, Demoly P. How can we better classify NSAID hypersensitivity reactions? - Validation from a large database. Int Arch Allergy Immunol 2012: 159: 306-12.

7. Ameratunga R, Randall N, Dalziel S, Anderson BJ. Samter's triad in childhood: a warning for those prescribing NSAIDs. Pediatr Anesth 2013: 23: 757-9.

8. Kowalski, M. L. and J. S. Makowska. Seven steps to the diagnosis of NSAIDs hypersensitivity: how to apply a new classification in real practice? Allergy Asthma Immunol Res 2015: 7: 312-20.

9. Demoly P, Kropf R, Bircher A, Pichler WJ. Drug hypersensitivity: questionnaire. Allergy 1999: 54: 999-1003.

10. Messaad D, Sahla H, Benahmed S, Godard P, Bousquet J, Demoly P. Drug provocation tests 
in patients with a history suggesting an immediate drug hypersensitivity reaction. Ann Intern Med 2004: 140: 1001-6.

11. Sampson HA, Muñoz-Furlong A, Campbell RL. Second symposium on the definition and management of anaphylaxis: summary report-Second National Institute of Allergy and Infectious Disease/Food Allergy and Anaphylaxis Network symposium. J Allergy Clin Immunol 2006: 117: 391-7.

12. Doña I, Blanca-López N, Torres MJ et al. NSAID-induced urticaria/angioedema does not evolve into chronic urticaria: a 12-year follow-up study. Allergy 2014: 69: 438-44.

13. Cavkaytar O, Arik Yilmaz E, Karaatmaca et al. Different Phenotypes of Non-Steroidal AntiInflammatory Drug Hypersensitivity during Childhood. Int Arch Allergy Immunol 2015: 167: 211-21.

14. Ayuso P, Blanca-López N, Doña I et al. Advanced phenotyping in hypersensitivity drug reactions to NSAIDs. Clin Exp Allergy 2013: 43: 1097-109.

15. Berges-Gimeno MP, Simon RA, Stevenson DD. The natural history and clinical characteristics of aspirin-exacerbated respiratory disease. Ann Allergy Asthma Immunol 2002: 89: 474-8.

16. Zambonino MA, Torres MJ, Muñoz C et al. Drug provocation tests in the diagnosis of hypersensitivity reactions to non-steroidal anti-inflammatory drugs in children. Pediatr Allergy Immunol 2013: 24: 151-9. 